\section{Is testosterone responsible for age differences in the susceptibility of cisplatin- induced nephrotoxicity in male C57BL/6 mice?}

\section{Editorial}

I read with interest a recently published article in the Age (Dordr) by Wen and colleagues, entitled "Aging increases the susceptibility of cisplatin-induced nephrotoxicity" [1]. The authors have concluded that the downregulation of efflux transporter, Multidrug and Toxin Extrusion Protein 1 (MATE1) and the decrease of cisplatin (CDDP) filtration responsible for aging promote CDDP-induced nephrotoxicity in male C57BL/6 mice [1]. Here, I would like to underline further potential possible mechanisms concerning this phenomenon. The Organic Cation Transporter 2 (OCT2) mediates CDDP-induced nephrotoxicity and act as target for protective interventions [2]. It has been demonstrated that OCT2 is the critical OCT isoform which is responsible for CDDP uptake in the kidney. Over expression of OCT2 in Human Embryonic Kidney (HEK293) cells increases CDDP uptake and cellular sensitivity to CDDP toxicity [3]. Gender differences of CDDP-induced nephrotoxicity may be related to CDDP uptake by OCT2. In addition, CDDP uptake was increased by OCT2 overexpression in male rats and was associated with increased cellular sensitivity to CDDP toxicity [4].

tOne of the possible mechanism regarding age-related differences in susceptibility to CDDP-induced renal toxicity in male rats may be related to CDDP uptake by OCT2 and the main determinant for that is testosterone [5]. This conclusion is in agreement with a study that showed renal OCT2 levels dramatically increased after day 25 in male rats and that due to an increase in testosterone levels before day 25 and renal OCT2 levels in gonadectomized adult male rats were substantially lower than that present in the kidneys from intact males [6]. Furthermore, another study demonstrated that CDDP was less likely to induce nephrotoxicity and had lower renal platinum concentrations in younger rats (age, 10 to 15 days) than that found in adult rats following CDDP exposure [7]. On the other hand, wen and colleagues used C57BL/6 mice (male, 3- and 18-monthold) to explore age differences in susceptibility to CDDP-induced nephrotoxicity and they found that the mRNA of OCT2, but not the protein level, was down regulated in aged mice [1]. An important factor that has to be considered when measuring sex hormones is the age of the animal [8]. Moreover, study of two groups of male C57BL/6 mice was found the mean testosterone levels higher in the older group rather than younger group [9]. Interestingly, study concluded that testosterone is responsible for enhanced susceptibility of male $\mathrm{C} 57 \mathrm{BL} / 6$ mice to ischemic renal injury by less activation of Nitric Oxide Synthases (NOSs), less activation of Akt and extracellular signal related kinase (ERK), greater activation of c-jun N-terminal kinase (JNK), greater expression of intracellular adhesion molecule-1 (ICAM-1), greater infiltration of leukocytes and more apoptosis [10]. Furthermore, several studies demonstrated that testosterone increase renin activity in male mice; which subsequent increase angiotensin II [11]. Angiotensin II has an impact on the substrate affinity of OCT2 to increase transporter rate by inducing conformational changes at the substrate binding sites [12]. Thus, I can conclude that testosterone plays critical role in increase affinity of CDDP uptake by OCT2 in mice. Finally, I would like to summarize the main determinant for aging increases the susceptibility of CDDP-induced nephrotoxicity in male $\mathrm{C} 57 \mathrm{BL} / 6$ mice is testosterone. In addition, decrease of MATE1 in old mice kidney can limit the efflux of CDDP, causing more CDDP renal accumulation and severe nephrotoxicity.

\section{EL Arabey $A A^{* 1}$}

${ }^{1}$ Department of Pharmacology and Toxicology, Al-Azhar University, Egypt ${ }^{*}$ Author for correspondence: Tel.:+00201006180922 ph.amrcapa@gmail.com 


\section{References}

1. Wen J, Zeng M, Shu Y et al. Aging increases the susceptibility of cisplatin-induced nephrotoxicity. AGE 37, 1-13 (2015).

2. Ciarimboli G, Deuster D, Knief A et al. Organic cation transporter 2 mediates cisplatin-induced oto-and nephrotoxicity and is a target for protective interventions. Am. J. Pathol. 176, 1169-1180 (2010).

3. Ciarimboli, G, Ludwig T, Lang D et al. Cisplatin nephrotoxicity is critically mediated via the human organic cation transporter 2. Am. J. Pathol. 167, 1477-1484 (2005).

4. EL Arabey AA. Gender Difference in CisplatinInduced Nephrotoxicity in a Rat Model. Nephrourol. Mon. 7 (2015).
5. El Arabey AA. Sex and Age Differences Related to Renal OCT2 Gene Expression in Cisplatininduced Nephrotoxicity. Iran. J. Kidney. Dis. 9, 335-336(2015).

6. Slitt AL, Cherrington NJ, Hartley DP et al. Tissue distribution and renal developmental changes in rat organic cation transporter mRNA levels. Drug. Metab. Dispos. 30, $212-219$ (2002).

7. Jongejan HTM, Provoost AP, Wolff ED et al. Nephrotoxicity of cis-platin comparing young and adult rats. Pediatric research 20, 9-14 (1986).

8. Jones RD, Pugh PJ, Hall J. Altered circulating hormone levels, endothelial function and vascular reactivity in the testicular feminised mouse. Eur. J. Endocrinol. 148, 111-120 (2003).

9. Brouillette J, Rivard K., Lizotte E. Sex and strain differences in adult mouse cardiac repolarization: importance of androgens. Cardiovasc. Res. 65, 148-157 (2005).

10. Park KM, Kim JI, Ahn Y et al. Testosterone is responsible for enhanced susceptibility of males to ischemic renal injury. J. Biol. Chem. 279, 52282-52292 (2004).

11. Catanzaro DF, Mesterovic NIKO, Morris BJ. Studies of the Regulation of Mouse Renin Genes by Measurement of Renin Messenger Ribonucleic Acid. 117, 872-878 (1985).

12. Guckel D, Ciarimboli G, Pavenstaedt H. Regulation of organic cation transport in isolated mouse proximal tubules involves complex changes in protein trafficking and substrate affinity. Cellular Physiology and Biochemistry 30, 269-281(2012). 